\title{
A DISCRETE FRACTIONAL GRONWALL INEQUALITY
}

\author{
RUI A. C. FERREIRA
}

(Communicated by Varghese Mathai)

\begin{abstract}
In this work we derive a Gronwall type inequality within the discrete fractional setting. Our results constitute generalizations on the inequalities of discrete calculus.
\end{abstract}

\section{INTRODUCTION}

One of the most important inequalities in the theory of differential equations is known as the Gronwall inequality. It was published in the year 1919 in the work by Gronwall 14. Since then many generalizations and extensions of this inequality became part of the literature [19]. The discrete Gronwall inequality seems to have appeared first in the work of Mikeladze [16] in the year 1935, and now it is used for example in proving convergence of the discrete variable methods for ordinary, partial as well as integral equations [1].

Difference equations arise as mathematical models describing many real life situations, e.g., queueing problems, electrical networks, economics, etc., and this is reason enough to explore such a theory. Nevertheless, perhaps due to the advent of computers, only relatively recently difference equations have started receiving the attention they deserve 1 and nowadays it is a discipline of mathematics by itself.

Much more recently, maybe due to the explosion in research within the fractional differential calculus (see for example the books [15, 18] ), the theory of discrete fractional calculus is being developed. Usually there are many definitions of derivatives within a certain fractional setting, and researchers use the ones that are more suitable in a determined context. Indeed, perhaps the first time that a definition of a discrete fractional derivative appeared in the literature was in the work by Díaz et al. 10 in the year 1974. This definition was given through an infinite sum, while later, Gray et al. [13, in 1988, presented a definition based on a finite sum. This latter definition uses what is called in the literature the nabla difference operator [we refer the reader to the recent work by Anastassiou [3] obtaining some inequalities (e.g., Opial, Ostrowski) using the fractional nabla operator].

In 1989, Miller et al. [17] defined a fractional sum of order $\alpha>0$ via the solution of a linear difference equation (cf. (2.1) below) and proved some basic properties of this operator. The calculus emerging from this definition has become appealing to many authors and now it is a matter of strong research, in various

Received by the editors December 27, 2010.

2010 Mathematics Subject Classification. Primary 26D15, 26A33; Secondary 39A12.

Key words and phrases. Discrete fractional calculus, Gronwall inequality, comparison theorem.

The author was supported by the Portuguese Foundation for Science and Technology (FCT) through the R\&D unit Center of Research and Development in Mathematics and Applications (CIDMA). 
directions: existence and uniqueness of solutions to discrete fractional equations [4, 5, 7, 12, modelling tumor growths 6], continuity of solutions with respect to initial conditions and also to the order $\alpha$ of the derivative [11, the Euler-Lagrange equation and Legendre's optimality condition for calculus of variations problems [8].

It is our aim in this work to contribute to the development of this theory, presenting the (fundamental) Gronwall inequality (we refer the reader to the works 9, 20, for the continuous Gronwall inequality). We also would like to mention that besides the inequality some other interesting identities were obtained (cf. for example equality (3.4) below).

This paper is organized as follows: In Section 2 we provide the reader the necessary background on discrete fractional calculus. In Section 3 we present our achievements, namely, a comparison theorem, the explicit solution of an initial value problem, the Gronwall inequality, as well as some consequences of all the results.

\section{Preliminaries on Discrete fractional CAlCulus}

In this section we introduce the reader to basic concepts and results about discrete fractional calculus.

Throughout this work and as usual, we assume that empty sums and products equal 0 and 1 , respectively.

The power function is defined by

$$
x^{(y)}=\frac{\Gamma(x+1)}{\Gamma(x+1-y)}, \text { for } x, x-y \in \mathbb{R} \backslash\{\ldots,-2,-1\} .
$$

Remark 2.1. Using the properties of the Gamma function it is easily seen that, for $x \geq y \geq 0$ we get $x^{(y)} \geq 0$.

For $a \in \mathbb{R}$ and $0<\alpha \leq 1$ we define the set $\mathbb{N}_{a}^{\alpha}=\{a+\alpha-1, a+\alpha, a+\alpha+$ $1, \ldots\}$. Also, we use the notation $\sigma(s)=s+1$ for the shift operator and $(\Delta f)(t)=$ $f(t+1)-f(t)$ for the forward difference operator.

For a function $f: \mathbb{N}_{a}^{1} \rightarrow \mathbb{R}$, the discrete fractional sum of order $\alpha \geq 0$ is defined as

$$
\begin{aligned}
\left({ }_{a} \Delta^{0} f\right)(t) & =f(t), \quad t \in \mathbb{N}_{a}^{1}, \\
\left({ }_{a} \Delta^{-\alpha} f\right)(t) & =\frac{1}{\Gamma(\alpha)} \sum_{s=a}^{t-\alpha}(t-\sigma(s))^{(\nu-1)} f(s), \quad t \in \mathbb{N}_{a}^{\alpha}, \alpha>0 .
\end{aligned}
$$

Remark 2.2. Note that the operator ${ }_{a} \Delta^{-\alpha}$ with $\alpha>0$ maps functions defined on $\mathbb{N}_{a}^{1}$ to functions defined on $\mathbb{N}_{a}^{\alpha}$. Also observe that if $\alpha=1$, then we get the summation operator

$$
\left({ }_{a} \Delta^{-1} f\right)(t)=\sum_{s=a}^{t-1} f(s) .
$$

The discrete fractional derivative of order $\alpha \in(0,1]$ is defined by

$$
\left({ }_{a} \Delta^{\alpha} f\right)(t)=\left(\Delta_{a} \Delta^{-(1-\alpha)} f\right)(t), \quad t \in \mathbb{N}_{a}^{\alpha} .
$$

Remark 2.3. Note that if $\alpha=1$, then the fractional derivative is just the forward difference operator. 
Lemma 2.4 (4 Lemma 2.3]). Suppose that $\mu, \mu+\nu \in \mathbb{R} \backslash\{\ldots,-2,-1\}$. Then,

$$
\left({ }_{\mu} \Delta^{-\nu} s^{(\mu)}\right)(t)=\frac{\Gamma(\mu+1)}{\Gamma(\mu+\nu+1)} t^{(\mu+\nu)}, \quad t \in \mathbb{N}_{\mu}^{\nu+1} .
$$

The following result is an immediate consequence of the previous lemma.

Corollary 2.5. Let $a \in \mathbb{R}$. Suppose that $\mu, \mu+\nu \in \mathbb{R} \backslash\{\ldots,-2,-1\}$. Then,

$$
\left({ }_{a} \Delta^{-\nu}(s-a+\mu)^{(\mu)}\right)(t)=\frac{\Gamma(\mu+1)}{\Gamma(\mu+\nu+1)}(t-a+\mu)^{(\mu+\nu)}, \quad t \in \mathbb{N}_{a}^{\nu+1} .
$$

We will make use of the following equality.

Theorem 2.6. Let $a, b$ be two real numbers such that $b=a+k$, with $k \in \mathbb{N}_{1}^{1}$. For $\mathbb{N}(a, b)=\{a, a+1, \ldots, b\}$ and a real-valued function $F(\tau, s)$ defined on $\mathbb{N}(a, b) \times$ $\mathbb{N}(a, b)$, the next equality holds:

$$
\sum_{\tau=a}^{b} \sum_{s=a}^{\tau-1} F(\tau, s)=\sum_{s=a}^{b} \sum_{\tau=s+1}^{b} F(\tau, s) .
$$

Proof. This result follows from [2, Theorem 10] by choosing the time scale $\mathbb{T}$ to be $\mathbb{T}=\mathbb{Z}$

Remark 2.7. We note that, by Theorem 2.6] it follows that

$$
\begin{aligned}
\sum_{\tau=a}^{b} \sum_{s=a}^{\tau} F(\tau, s) & =\sum_{\tau=a}^{b+1} \sum_{s=a}^{\tau-1} F(\tau-1, s) \\
& =\sum_{s=a}^{b+1} \sum_{\tau=s+1}^{b+1} F(\tau-1, s) \\
& =\sum_{s=a}^{b+1} \sum_{\tau=s}^{b} F(\tau, s) \\
& =\sum_{s=a}^{b} \sum_{\tau=s}^{b} F(\tau, s) .
\end{aligned}
$$

\section{MAIN RESUlts}

We start by proving a comparison result for the fractional summation operator.

Theorem 3.1. Let $a \in \mathbb{R}, 0<\alpha \leq 1$ and $f(t, x): \mathbb{N}_{a}^{\alpha} \times \mathbb{R} \rightarrow \mathbb{R}$ be nondecreasing in $x$ for each $t \in \mathbb{N}_{a}^{\alpha}$. If $v, w: \mathbb{N}_{a}^{\alpha} \rightarrow \mathbb{R}$ are functions satisfying

$$
\begin{aligned}
& w(t) \geq \frac{A}{\Gamma(\alpha)}(t-a)^{(\alpha-1)}+\left({ }_{a} \Delta^{-\alpha} f(s+\alpha-1, w(s+\alpha-1))\right)(t), \quad t \in \mathbb{N}_{a}^{\alpha}, \\
& v(t) \leq \frac{B}{\Gamma(\alpha)}(t-a)^{(\alpha-1)}+\left({ }_{a} \Delta^{-\alpha} f(s+\alpha-1, v(s+\alpha-1))\right)(t), \quad t \in \mathbb{N}_{a}^{\alpha},
\end{aligned}
$$

where $A \geq B$ are two real numbers, then $w(t) \geq v(t)$ for all $t \in \mathbb{N}_{a}^{\alpha}$. 
Proof. Suppose that there exists $t_{0} \in \mathbb{N}_{a}^{\alpha}$ such that $w\left(t_{0}\right)<v\left(t_{0}\right)$. Define the number $t_{1}=\min \left\{t \in \mathbb{N}_{a}^{\alpha}: w(t)<v(t)\right\}$. Then,

$$
\begin{aligned}
& w\left(t_{1}\right) \geq \frac{A}{\Gamma(\alpha)}\left(t_{1}-a\right)^{(\alpha-1)}+\frac{1}{\Gamma(\alpha)} \sum_{s=a}^{t_{1}-\alpha}\left(t_{1}-\sigma(s)\right)^{(\alpha-1)} f(s+\alpha-1, w(s+\alpha-1)), \\
& v\left(t_{1}\right) \leq \frac{B}{\Gamma(\alpha)}\left(t_{1}-a\right)^{(\alpha-1)}+\frac{1}{\Gamma(\alpha)} \sum_{s=a}^{t_{1}-\alpha}\left(t_{1}-\sigma(s)\right)^{(\alpha-1)} f(s+\alpha-1, v(s+\alpha-1)),
\end{aligned}
$$

from which it follows that (with $\bar{s}=s+\alpha-1$ )

$$
\begin{aligned}
w\left(t_{1}\right)-v\left(t_{1}\right) \geq \frac{A-B}{\Gamma(\alpha)}\left(t_{1}-a\right)^{(\alpha-1)} & \\
& \quad+\frac{1}{\Gamma(\alpha)} \sum_{s=a}^{t_{1}-\alpha}\left(t_{1}-\sigma(s)\right)^{(\alpha-1)}[f(\bar{s}, w(\bar{s}))-f(\bar{s}, v(\bar{s}))]
\end{aligned}
$$

$\geq 0$.

This is a contradiction, and therefore the theorem is proved.

In [5] the following initial value problem was studied:

$$
\begin{aligned}
\left(\alpha_{\alpha-1} \Delta^{\alpha} x\right)(t) & =f(t+\alpha-1, x(t+\alpha-1)), \quad t \in\{0,1,2, \ldots\}, \\
x(\alpha-1) & =x_{0},
\end{aligned}
$$

where $\alpha \in(0,1], f$ is a real-valued function and $x_{0}$ is a real number. The unique solution implicitly obtained was

$$
x(t)=\frac{t^{(\alpha-1)}}{\Gamma(\alpha)} x_{0}+\frac{1}{\Gamma(\alpha)} \sum_{s=0}^{t-\alpha}(t-\sigma(s))^{(\alpha-1)} f(s+\alpha-1, x(s+\alpha-1)), \quad t \in \mathbb{N}_{0}^{\alpha} .
$$

Following the same procedure, it is easy to obtain

$$
x(t)=\frac{(t-a)^{(\alpha-1)}}{\Gamma(\alpha)} x_{a}+\frac{1}{\Gamma(\alpha)} \sum_{s=a}^{t-\alpha}(t-\sigma(s))^{(\alpha-1)} f(s+\alpha-1, x(s+\alpha-1)), \quad t \in \mathbb{N}_{a}^{\alpha},
$$

as the solution of the following nonlinear fractional difference initial value problem:

$$
\begin{aligned}
\left(a+\alpha-1 \Delta^{\alpha} x\right)(t) & =f(t+\alpha-1, x(t+\alpha-1)), \quad t \in\{a, a+1, a+2, \ldots\}, \\
x(a+\alpha-1) & =x_{a} .
\end{aligned}
$$

Now we will solve (3.1) in the particular case when $f(t, x)=y(t) x$. The next result together with Theorem 3.1 will give us the desired Gronwall type inequality.

Theorem 3.2. Let $a \in \mathbb{R}$ and $\alpha \in(0,1]$. Suppose that $y: \mathbb{N}_{a}^{\alpha} \rightarrow \mathbb{R}$ is a function. Define an operator $T$ by

$$
\begin{aligned}
\left(T_{y}^{0} f\right)(t) & =f(t), \\
\left(T_{y}^{1} f\right)(t) & =\left(T_{y} f\right)(t)=\left({ }_{a} \Delta^{-\alpha} y(s+\alpha-1) f(s+\alpha-1)\right)(t), \\
\left(T_{y}^{k+1} f\right)(t) & =\left(T_{y} T_{y}^{k}\right)(t), \quad k \in \mathbb{N}_{1}^{1},
\end{aligned}
$$

for $t \in \mathbb{N}_{a}^{\alpha}$. Then the function

$$
x(t)=\frac{x_{a}}{\Gamma(\alpha)} \sum_{k=a}^{t-(\alpha-1)}\left(T_{y}^{k-a}(s-a)^{(\alpha-1)}\right)(t), \quad t \in \mathbb{N}_{a}^{\alpha},
$$


is the solution of the summation equation

$$
x(t)=\frac{(t-a)^{(\alpha-1)}}{\Gamma(\alpha)} x_{a}+\left({ }_{a} \Delta^{-\alpha} y(s+\alpha-1) x(s+\alpha-1)\right)(t), \quad t \in \mathbb{N}_{a}^{\alpha} .
$$

Proof. Fix $t \in \mathbb{N}_{a}^{\alpha}$. We have that

$$
\begin{aligned}
& \frac{(t-a)^{(\alpha-1)}}{\Gamma(\alpha)} x_{a}+\left({ }_{a} \Delta^{-\alpha} y(s+\alpha-1) x(s+\alpha-1)\right)(t) \\
& =\frac{(t-a)^{(\alpha-1)}}{\Gamma(\alpha)} x_{a}+\frac{1}{\Gamma(\alpha)} \sum_{s=a}^{t-\alpha}(t-\sigma(s))^{(\alpha-1)} y(s+\alpha-1) x(s+\alpha-1) \\
& =\frac{(t-a)^{(\alpha-1)}}{\Gamma(\alpha)} x_{a} \\
& +\frac{1}{\Gamma(\alpha)} \sum_{s=a}^{t-\alpha}(t-\sigma(s))^{(\alpha-1)} y(s+\alpha-1) \frac{x_{a}}{\Gamma(\alpha)} \sum_{k=a}^{s}\left(T_{y}^{k-a}(r-a)^{(\alpha-1)}\right)(s+\alpha-1) \\
& =\frac{(t-a)^{(\alpha-1)}}{\Gamma(\alpha)} x_{a} \\
& +\frac{x_{a}}{\Gamma(\alpha)} \sum_{s=a}^{t-\alpha} \frac{1}{\Gamma(\alpha)} \sum_{k=a}^{s}(t-\sigma(s))^{(\alpha-1)} y(s+\alpha-1)\left(T_{y}^{k-a}(r-a)^{(\alpha-1)}\right)(s+\alpha-1) \\
& =\frac{(t-a)^{(\alpha-1)}}{\Gamma(\alpha)} x_{a} \\
& +\frac{x_{a}}{\Gamma(\alpha)} \sum_{k=a}^{t-\alpha} \frac{1}{\Gamma(\alpha)} \sum_{s=k}^{t-\alpha}(t-\sigma(s))^{(\alpha-1)} y(s+\alpha-1)\left(T_{y}^{k-a}(r-a)^{(\alpha-1)}\right)(s+\alpha-1) \\
& =\frac{(t-a)^{(\alpha-1)}}{\Gamma(\alpha)} x_{a}+\frac{x_{a}}{\Gamma(\alpha)} \sum_{k=a}^{t-\alpha}\left(T_{y}^{k+1-a}(r-a)^{(\alpha-1)}\right)(t) \\
& =\frac{x_{a}}{\Gamma(\alpha)} \sum_{k=a}^{t-(\alpha-1)}\left(T_{y}^{k-a}(r-a)^{(\alpha-1)}\right)(t)=x(t),
\end{aligned}
$$

where the fourth equality follows by Remark 2.7 .

Corollary 3.3. If $y(t)=c$ for some $c \in \mathbb{R}$ and all $t \in \mathbb{N}_{a}^{\alpha}$ in Theorem 3.2 , then the solution given by (3.2) is

$$
x(t)=x_{a} \sum_{k=a}^{t-(\alpha-1)} \frac{c^{k-a}}{\Gamma((k-a) \alpha+\alpha)}(t-a+(k-a)(\alpha-1))^{((k-a) \alpha+\alpha-1)}, \quad t \in \mathbb{N}_{a}^{\alpha} .
$$

Proof. We only need to prove that, for a given $t \in \mathbb{N}_{a}^{\alpha}$,

$$
\left(T_{c}^{k-a}(s-a)^{(\alpha-1)}\right)(t)=\Gamma(\alpha) \frac{c^{k-a}}{\Gamma((k-a) \alpha+\alpha)}(t-a+(k-a)(\alpha-1))^{((k-a) \alpha+\alpha-1)},
$$

for all $k \in\{a, a+1, \ldots, t-(\alpha-1)\}$. We start by fixing $t \in \mathbb{N}_{a}^{\alpha} \backslash\{a+\alpha-1\}$ (the case $t=a+\alpha-1$ is clear) and then we use induction on $k$.

If $k=a$, then the equality in (3.3) is obvious. Assume now that the equality in (3.3) is true for some $k \in\{a, a+1, \ldots, t-1-(\alpha-1)\}$. Then, using Corollary 2.5] 
we get

$$
\begin{aligned}
& \left(T_{c}^{k+1-a}(s-a)^{(\alpha-1)}\right)(t)=\left(T_{c} T_{c}^{k-a}(s-a)^{(\alpha-1)}\right)(t) \\
& =\left(T_{c} \Gamma(\alpha) \frac{c^{k-a}}{\Gamma((k-a) \alpha+\alpha)}(s-a+(k-a)(\alpha-1))^{((k-a) \alpha+\alpha-1)}\right)(t) \\
& =\Gamma(\alpha) \frac{c^{k-a}}{\Gamma((k-a) \alpha+\alpha)}\left({ }_{k} \Delta^{-\alpha} c(s+\alpha-1-a+(k-a)(\alpha-1))^{((k-a) \alpha+\alpha-1)}\right)(t) \\
& =\Gamma(\alpha) \frac{c^{k+1-a}}{\Gamma((k-a) \alpha+\alpha)}\left({ }_{k} \Delta^{-\alpha}(s-k+(k-a) \alpha+\alpha-1)^{((k-a) \alpha+\alpha-1)}\right)(t) \\
& =\Gamma(\alpha) \frac{c^{k+1-a}}{\Gamma((k-a) \alpha+\alpha)} \frac{\Gamma((k-a) \alpha+\alpha)}{\Gamma((k-a) \alpha+\alpha+\alpha)}(t-k+(k-a) \alpha+\alpha-1)^{((k-a) \alpha+\alpha+\alpha-1)} \\
& =\Gamma(\alpha) \frac{c^{k+1-a}}{\Gamma((k+1-a) \alpha+\alpha)}\left(t-a+(k+1-a)(\alpha-1)^{((k+1-a) \alpha+\alpha-1)}\right.
\end{aligned}
$$

which proves the claim.

Remark 3.4. For $a=0$, Corollary 3.3 was previously obtained in [5] (see the equality in (3.7) therein). However, there is an inconsistency in that formula; i.e., instead of getting

$$
x(t)=x_{0} \sum_{k=0}^{t-(\alpha-1)} \frac{c^{k}}{\Gamma(k \alpha+\alpha)}(t+k(\alpha-1))^{(k \alpha+\alpha-1)},
$$

they obtained

$$
x(t)=x_{0} \sum_{k=0}^{t-(\alpha-1)} \frac{c^{k}}{\Gamma(k \alpha+\alpha)}(t+(k-1)(\alpha-1))^{(k \alpha+\alpha-1)} .
$$

We now present the Gronwall inequality within the discrete fractional setting.

Theorem 3.5 (Gronwall's inequality). Let $\alpha \in(0,1]$ and $a, d \in \mathbb{R}$. Suppose that $u, y: \mathbb{N}_{a}^{\alpha} \rightarrow \mathbb{R}$ are two functions with $y$ nonnegative. If

$$
u(t) \leq \frac{(t-a)^{(\alpha-1)}}{\Gamma(\alpha)} d+\left({ }_{a} \Delta^{-\alpha} y(s+\alpha-1) u(s+\alpha-1)\right)(t), \quad t \in \mathbb{N}_{a}^{\alpha},
$$

then

$$
u(t) \leq \frac{d}{\Gamma(\alpha)} \sum_{k=a}^{t-(\alpha-1)}\left(T_{y}^{k-a}(s-a)^{(\alpha-1)}\right)(t), \quad t \in \mathbb{N}_{a}^{\alpha} .
$$

Proof. Let $x$ be the solution of the equation

$$
x(t)=\frac{(t-a)^{(\alpha-1)}}{\Gamma(\alpha)} d+\left({ }_{a} \Delta^{-\alpha} y(s+\alpha-1) x(s+\alpha-1)\right)(t),
$$

which, by Theorem 3.2 , is

$$
x(t)=\frac{d}{\Gamma(\alpha)} \sum_{k=a}^{t-(\alpha-1)}\left(T_{y}^{k-a}(s-a)^{(\alpha-1)}\right)(t) .
$$

Since $y$ is nonnegative, then $f(t, x)=y(t) x$ is nondecreasing in $x$. Now an application of Theorem 3.1 immediately yields $u(t) \leq x(t)$. 
Let us now show that Theorem 3.5 is truly a generalization of the discrete Gronwall inequality. The next result can be found, e.g., in [1, Corollary 4.1.2].

Corollary 3.6. Suppose that $\alpha=1$ in Theorem 3.5. Then,

$$
u(t) \leq d+\sum_{s=a}^{t-1} y(s) u(s), \quad t \in \mathbb{N}_{a}^{1}=\{a, a+1, \ldots,\},
$$

implies

$$
u(t) \leq d \prod_{s=a}^{t-1}(1+y(s)), \quad t \in \mathbb{N}_{a}^{1} .
$$

Proof. We only have to show the validity of the following equality:

$$
\sum_{k=a}^{t}\left(T_{y}^{k-a} 1\right)(t)=\prod_{s=a}^{t-1}(1+y(s)), \quad t \in \mathbb{N}_{a}^{1} .
$$

To do that we start with two important observations:

$$
\begin{aligned}
\left(T_{y}^{t+1-a} 1\right)(t) & =0, \text { for all } t \in \mathbb{N}_{a}^{1} ; \\
\left(T_{y}^{k-a} 1\right)(t+1) & =\left(T_{y}^{k-a} 1\right)(t)+y(t)\left(T_{y}^{k-1-a} 1\right)(t), k \in\{a+1, a+2, \ldots, t+1\} .
\end{aligned}
$$

Now we use induction on $t$. The case $t=a$ is obvious; therefore, assume that (3.4) holds for $t \in \mathbb{N}_{a}^{1}$. Then,

$$
\begin{aligned}
\sum_{k=a}^{t+1}\left(T_{y}^{k-a} 1\right)(t+1) & =1+\sum_{k=a+1}^{t+1}\left(T_{y}^{k-a} 1\right)(t+1) \\
& =1+\sum_{k=a+1}^{t+1}\left[\left(T_{y}^{k-a} 1\right)(t)+y(t)\left(T_{y}^{k-1-a} 1\right)(t)\right] \\
& =\sum_{k=a}^{t}\left(T_{y}^{k-a} 1\right)(t)+y(t) \sum_{k=a}^{t}\left(T_{y}^{k-a} 1\right)(t) \\
& =(1+y(t)) \prod_{s=a}^{t-1}(1+y(s)) \\
& =\prod_{s=a}^{t}(1+y(s)) .
\end{aligned}
$$

The proof is done.

Remark 3.7. We observe that, since the solution of (3.1) is unique, then Corollary 3.3 together with equality (3.4) implies that (obviously with $y(t)=c$ )

$$
d \sum_{k=a}^{t} \frac{c^{k-a}}{\Gamma((k-a)+1)}(t-a)^{(k-a)}=d \prod_{s=a}^{t-1}(1+c) .
$$

Choosing $a=0$ and $d \neq 0$ one obtains

$$
\sum_{k=0}^{t} \frac{c^{k}}{\Gamma(k+1)} t^{(k)}=(1+c)^{t}, \quad t \in\{0,1,2, \ldots\},
$$


which is the same as

$$
\sum_{k=0}^{t}\left(\begin{array}{l}
t \\
k
\end{array}\right) c^{k}=(1+c)^{t}, \quad t \in\{0,1,2, \ldots\} ;
$$

i.e., a particular case of the binomial theorem is obtained.

\section{REFERENCES}

1. R. P. Agarwal, Difference equations and inequalities, second edition, Monographs and Textbooks in Pure and Applied Mathematics, 228, Dekker, New York, 2000. MR.1740241 (2001f:39001)

2. E. Akin, Cauchy functions for dynamic equations on a measure chain, J. Math. Anal. Appl. 267 (2002), no. 1, 97-115. MR.1886819 (2002k:34005)

3. G. A. Anastassiou, Nabla discrete fractional calculus and nabla inequalities, Math. Comput. Modelling 51 (2010), no. 5-6, 562-571. MR2594707 (2011a:26007)

4. F. M. Atici and P. W. Eloe, A transform method in discrete fractional calculus, Int. J. Difference Equ. 2 (2007), no. 2, 165-176. MR2493595 (2010a:26008)

5. F. M. Atici and P. W. Eloe, Initial value problems in discrete fractional calculus, Proc. Amer. Math. Soc. 137 (2009), no. 3, 981-989. MR2457438 (2009g:39037)

6. F. M. Atici and S. Şengül, Modeling with fractional difference equations, J. Math. Anal. Appl., 369 (2010), no. 1, 1-9. MR2643839 (2011c:39002)

7. F. M. Atici, P. W. Eloe, Two-point boundary value problems for finite fractional difference equations, J. Differ. Equ. Appl. 17 (2011), 445-456. MR2783359

8. N. R. O. Bastos, R. A. C. Ferreira and D. F. M. Torres, Necessary optimality conditions for fractional difference problems of the calculus of variations, Discrete Contin. Dyn. Syst. 29 (2011), no. 2, 417-437. MR2728463(2011j:49044)

9. Z. Denton and A. S. Vatsala, Fractional integral inequalities and applications, Comput. Math. Appl. 59 (2010), no. 3, 1087-1094. MR2579473 (2011a:26009)

10. J. B. Díaz and T. J. Osler, Differences of fractional order, Math. Comp. 28 (1974), 185-202. MR0346352(49:11077)

11. C. S. Goodrich, Continuity of solutions to discrete fractional initial value problems, Comput. Math. Appl. 59 (2010), no. 11, 3489-3499. MR2646320 (2011e:39002)

12. C. S. Goodrich, Solutions to a discrete right-focal fractional boundary value problem, Int. J. Difference Equ. 5 (2010), 195-216. MR2771325

13. H. L. Gray and N. F. Zhang, On a new definition of the fractional difference, Math. Comp. 50 (1988), no. 182, 513-529. MR 929549 (89i:39008)

14. T. H. Gronwall, Note on the derivatives with respect to a parameter of the solutions of a system of differential equations, Ann. of Math. (2) 20 (1919), no. 4, 292-296. MR1502565

15. R. L. Magin, Fractional Calculus in Bioengineering, Begell House, 2006.

16. Mikeladze, Sh.E., De la résolution numérique des équations intégrales, Bull. Acad. Sci. URSS VII (1935), 255-257 (in Russian).

17. K. S. Miller and B. Ross, Fractional difference calculus, in Univalent functions, fractional calculus, and their applications (Kōriyama, 1988), 139-152, Horwood, Chichester, 1989. MR1199147 (93h:26010)

18. K. S. Miller and B. Ross, An introduction to the fractional calculus and fractional differential equations, Wiley, New York, 1993. MR1219954 (94e:26013)

19. B. G. Pachpatte, Integral and finite difference inequalities and applications, Mathematics Studies 205, Elsevier, 2006. MR2286291(2008a:26003)

20. H. Ye, J. Gao and Y. Ding, A generalized Gronwall inequality and its application to a fractional differential equation, J. Math. Anal. Appl. 328 (2007), no. 2, 1075-1081. MR2290034

Department of Mathematics, Lusophone University of Humanities and Technologies, 1749-024 Lisbon, Portugal

E-mail address: ruiacferreira@ulusofona.pt 